\title{
Optical pulsations of the Crab nebula pulsar with AquEYE
}

\section{Germanà ${ }^{* \dagger}$}

Department of Astronomy, University of Padua

INAF - Astronomical Observatory of Padova

E-mail: claudio.germana@unipd.it

\section{Zampieri}

INAF - Astronomical Observatory of Padova

E-mail: luca.zampieri@oapd.inaf.it

\section{Capraro}

Department of Information Engineering, University of Padua

E-mail: ivan.capraro@dei.unipd.it

\section{Facchinetti}

Department of Astronomy, University of Padua

E-mail: claudia.facchinetti@unipd.it

\section{G. Naletto}

Department of Information Engineering, University of Padua CNR/INFM/LUXOR Padova

E-mail: naletto@dei.unipd.it

\section{T. Occhipinti}

Department of Information Engineering, University of Padua

E-mail: tommaso.occhipintiddei.unipd.it

\section{E. Verroi}

Department of Information Engineering, University of Padua

E-mail: verroi@dei.unipd.it

\section{P. Zoccarato}

Department of Astronomy, University of Padua

CISAS - Center of Studies and Activities for Space "G. Colombo", University of Padova

E-mail: paolo.zoccarato@unipd.it

\section{and C. Barbieri}

Department of Astronomy, University of Padua

E-mail: cesare.barbieri@unipd.it 
As is well known, the Crab pulsar displays a light curve with a characteristic double peak profile having a period of 33 milliseconds, almost aligned in phase over the whole electromagnetic spectrum. Since it is the brightest pulsar in the optical band $(V \sim 16)$, its pulse profile has been extensively monitored by several authors; it is also used as a standard candle to calibrate fluxes and for testing instruments with high time resolution.

We have studied the optical light curve of the Crab pulsar by means of a novel photon counting instrument (dubbed AquEYE, the Asiago Quantum EYE), that has the capability to provide data with exceptionally high temporal resolution and time tagging accuracy of each incoming photon.

Polarimetry days in Rome: Crab status, theory and prospects October 16-17 2008

Rome, Italy

* Speaker.

${ }^{\dagger}$ Aknowledgements: This study was partly funded by University of Padua, MIUR, and Fondazione CARIPARO. We thank A. Possenti for the kind help with Tempo2. We thank also INAF and the Galileo Supervising Authority for providing support. 


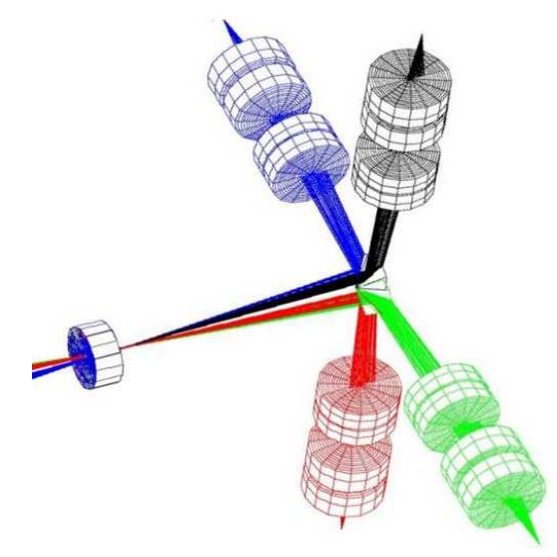

Figure 1: Optical design of AquEYE (see text).

\section{Optical studies of the Crab Nebula pulsar}

The pulsating neutron star (PSR B0531+21) hosted in the Crab Nebula has been extensively observed in several energy bands. The first pulsating signal ever detected was in the radio band (Staeilin \& Reifeinstein 1968). Very soon, a counterpart was discovered in the optical band (Cocke et al. 1969; Lynds et al. 1969). Thereafter optical data have been obtained by several groups (see for instance Karpov et al. 2007), with the aim of acquiring knowledge about the physical properties of a young fast rotating neutron star from its light curve.

By means of a stroboscopic instrument, Čadež and Galičič (1996) found a 60 s modulation in both phase and amplitude of the optical light curve of the Crab. This raised the question whether a young fast rotating neutron star can freely precess (Čadež et al. 1997). If confirmed, free precession can help us to constrain the equation of state of a neutron star.

Studies of the polarization of the optical signal were also performed by means of a highspeed photo-polarimeter (Kanbach et al. 2005). With this instrument, it was possible to study the polarization of both pulse and off-pulse emission separately. Such studies are useful to understand the physical properties of the magnetosphere around the neutron star.

\section{AquEYE: the Asiago Quantum Eye}

The optical design of AquEYE follows that of a previous study for the ESO E-ELT, QuantEYE (Barbieri et al. 2007, 2008). As shown in Fig.1, the pupil of the telescope is divided into four subpupils by means of a pyramidal mirror. Suitable lens systems collimate each beam sections, where broad and narrow band filters can be inserted. Then each sub-pupil is connected to a dedicated Single Photon Avalanche Diode (SPAD). A system of four independent fast photon-counters is created. The advantage of this design is to partly recover dead time effects in each SPAD, to increase the sustainable count rate, and to allow cross-correlations among the different sub-pupils. 


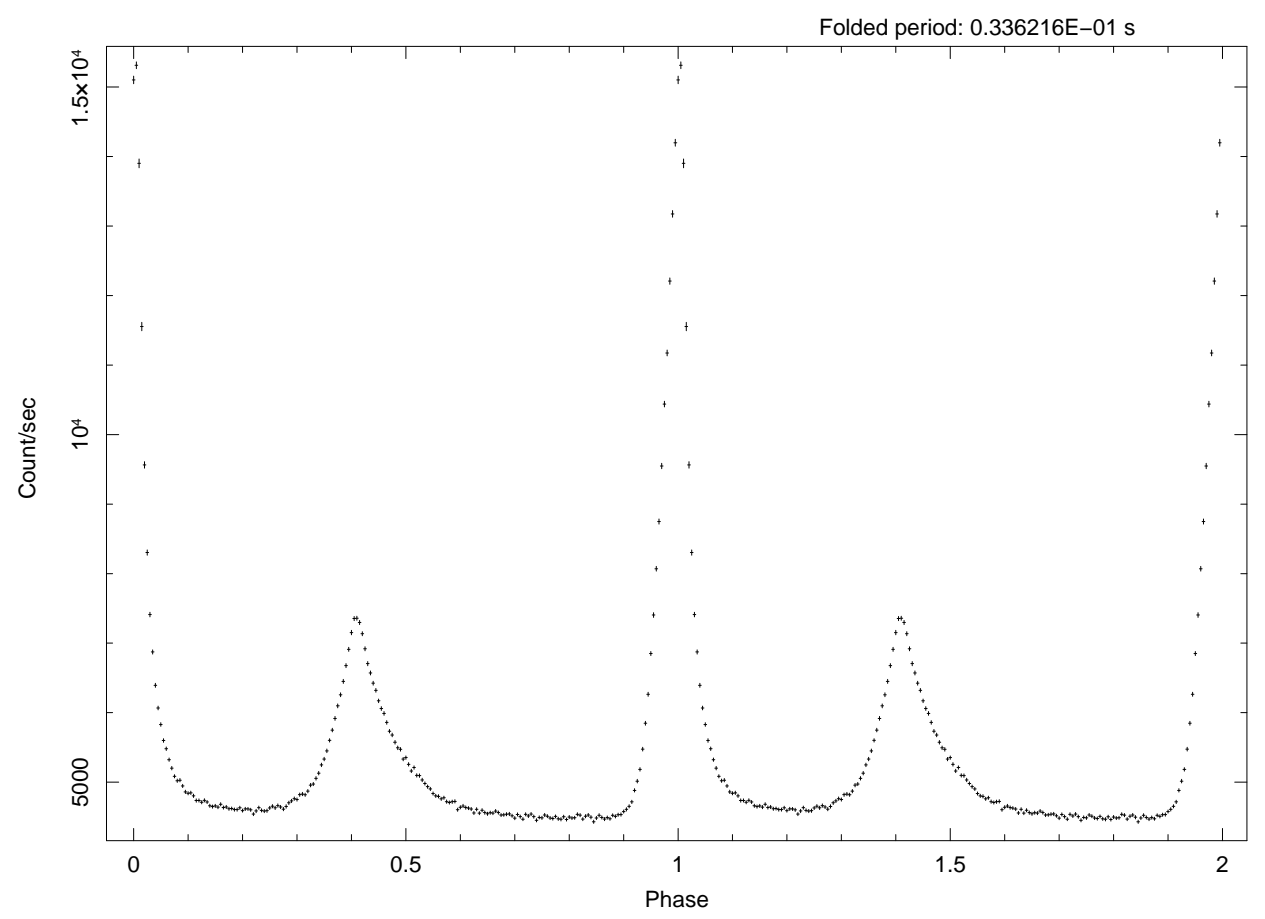

Start Time 14750 1:49:06:675 Stop Time 14750 2:19:04:172

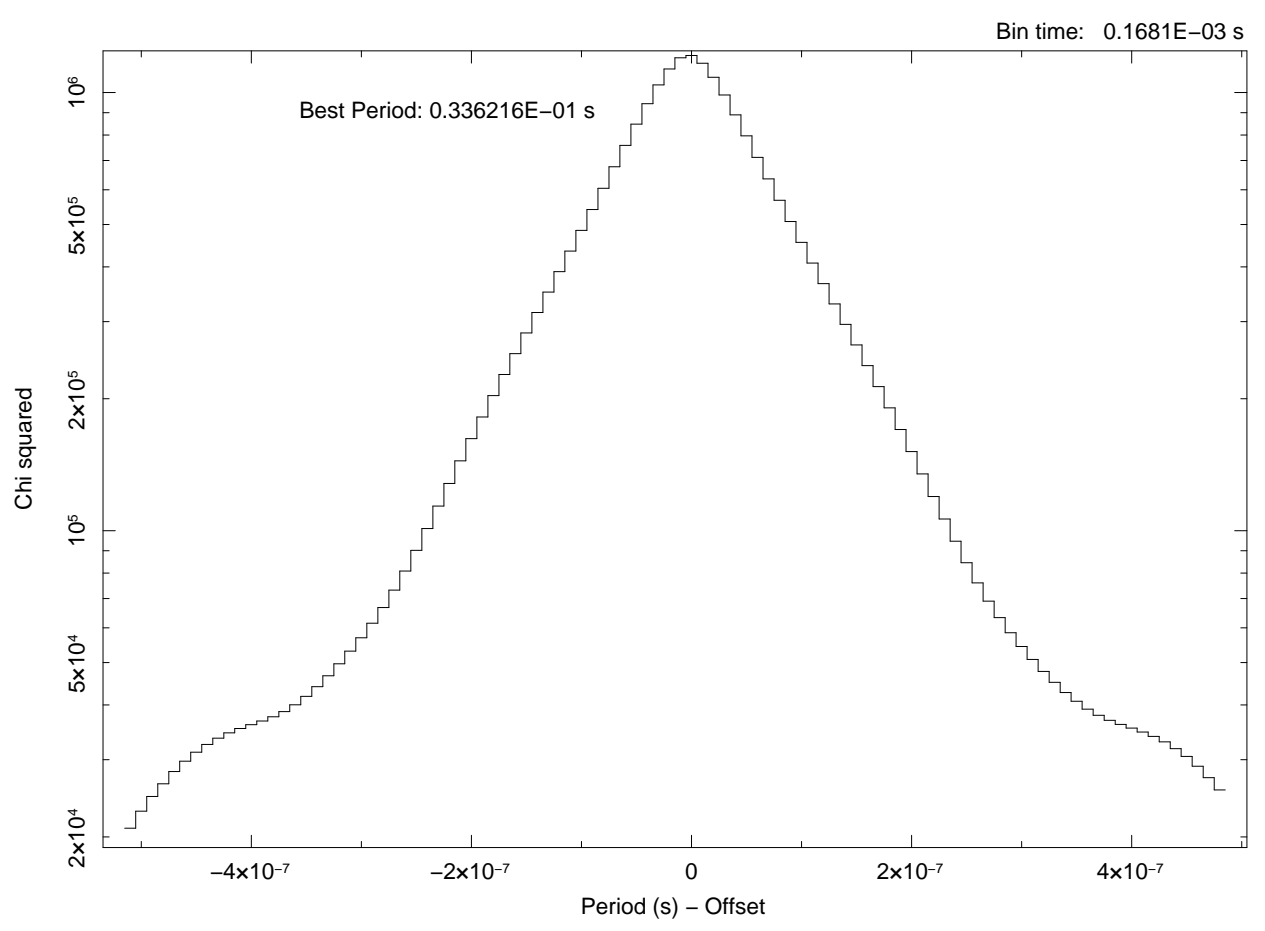

Start Time 14750 1:49:06:675 Stop Time 14750 2:19:04:172

Figure 2: Folded light curve (top) and best-period (bottom) of the Crab pulsar measured by AquEYE. 


\subsection{Optical light curve of the Crab pulsar with AquEYE}

Fig.2 shows an example of the folded light curve of the Crab pulsar obtained with AquEYE mounted at the $182 \mathrm{~cm}$ telescope of Cima Ekar (Asiago). The time series is 30 minutes long and was obtained on October 11, 2008. The phase binning time is $\sim 168 \mu \mathrm{sec}$ (200 bins per phase). Corrections for the background emission were not performed. Only a barycentric correction on the photon arrival time was applied. Observations were made in collaboration with the research group headed by Prof. Andrej Čadež of the Department of Physics, University of Ljubljana.

\subsection{Period of the pulsar measured by AquEYE}

Fig. 2 shows the best period of the Crab pulsar as measured by AquEYE (the start and stop of observation is expressed in "Truncated Julian Day", the time is in UTC). The value of this best period at the barycenter of the solar system on October 11, 2008 is $33.6216 \mathrm{~ms}$, with an estimated error of $100 \mathrm{~ns}$ ( $1 \sigma$ confidence). Data were processed with the task efsearch of the timing analysis software Xronos (http://xronos.gsfc.nasa.gov/). The period from the radio ephemeris at the same epoch is $33.621638 \mathrm{~ms}$, in agreement with our measurement within the errors. Further refinement is expected from the application to our data of the Tempo2 software (http://www.atnf.csiro.au/research /pulsar/ppta/tempo2), now in progress.

\section{Future topics: Second order interferometry}

Extremely high spatial and temporal resolutions, in particular for hot stars, insensitive to telescope optical quality as well as to atmospheric flickering, are feasible with second-order intensity interferometry. One of our goals is to improve this technique with both Extremely Large Telescopes and QuantEYE.

The spatial-intensity interferometry was pioneered by Hanbury Brown and Twiss (The Intensity Interferometer, Hanbury Brown 1974), and now receives a novel attention. The stellar intensity interferometer may be considered as the first experiment in quantum optics. The second-order spatial coherence of light is measured from the correlation of the nanosecond-scale fluctuations observed in the photon fluxes of two telescopes, thus deducing angular widths of stars.

Photon-correlation spectroscopy is the equivalent in the time domain of the spatial-intensity interferometry. Intensity fluctuations are correlated over baselines (delays) in time. From the measured temporal coherence, the spectral linewidth is deduced. The method has been now proposed to seek cosmic lasers (Dravins and Germanà 2008). Laser spectral lines are expected to be very narrow (FWHM $<1 \mathrm{~m} \AA$ ), requiring a spectral resolution $R \sim 10^{8}$ much higher than that reachable with conventional spectrographs (Johansson and Letokhov 2007). 


\section{References}

[1] Barbieri, C., Naletto, G., Occhipinti, T., Tamburini, F., Giro, E., D’Onofrio, M., Sain, E., \& Zaccariotto, M. 2007, Memorie della Societa Astronomica Italiana Supplement, 11, 190

[2] Barbieri, C., Naletto, G., Occhipinti, T., Facchinetti, C., Verroi, E., Giro, E., Di Paola, A., et al. 2008, Journal of Modern Optics, in press

[3] Čadež, A., \& Galičič, M. 1996, A\&A, 306, 443

[4] Čadež, A., Galičič, M., \& Calvani, M. 1997, A\&A, 324, 1005

[5] Cocke, W. J., Disney, M. J., \& Taylor, D. J. 1969, Nat, 221, 525

[6] Dravins, D., \& Germanà, C. 2008, in High Time Resolution Astrophysics: The Universe at Sub-Second Timescales, ed. O. Ryan, A. Shearer, AIP conf.proc., 984, 216

[7] Hanbury Brown, R., 1974, The Intensity Interferometer

[8] Johansson, S., \& Letokhov, V. S. 2007, New Astronomy Review, 51, 443

[9] Kanbach, G., Słowikowska, A., Kellner, S., \& Steinle, H. 2005, Astrophysical Sources of High Energy Particles and Radiation, 801, 306

[10] Karpov, S., Beskin, G., Biryukov, A., Plokhotnichenko, V., Debur, V., \& Shearer, A. 2007, arXiv:0709.2580

[11] Lynds, R., Maran, S. P., \& Trumbo, D. E. 1969, ApJ, 155, L121

[12] Staelin, D. H., \& Reifenstein, E. C. 1968, Science, 162, 1481 